\title{
Expanding Nurse Practitioner Scope of Practice in Michigan Effects on Health Care Delivery
}

S ome forecasts have pointed to a looming physician shortage in the United States, particularly in light of growing demand for health services from the Affordable Care Act and a rising elderly population. One strategy for addressing this issue is to allow nurse practitioners (NPs) to have "full scope of practice" - that is, to autonomously deliver care that falls within their educational preparation: diagnosing and treating conditions, writing prescriptions, and performing minor procedures. Many states have done this. In the state of Michigan, the legislature is considering how to define NP scope of practice. NPs in the state currently function under the same rules as registered nurses, limiting the care that they can provide to patients without the oversight of physicians. Would a more expansive scope of practice for NPs improve health care for the residents of Michigan?

To address this question, a team of RAND researchers examined the peer-reviewed literature on the effect of expanded NP scope of practice regulations on several health delivery outcomes: the supply of providers, access to care, utilization of care, costs, quality, and patient outcomes. Researchers used strict criteria to identify only empirically rigorous studies, leading them to eliminate a substantial share of the published literature in this area. They then used results from the literature review to estimate Michigan-specific impacts of full NP scope of practice. The study was commissioned by the Michigan Council of Nurse Practitioners.

\section{Results: Access, Utilization, and Patient- Centeredness Could Possibly Improve}

Provider supply: A small number of studies suggest that allowing full NP scope of practice increases the supply of providers, but the data were insufficient to estimate specific impacts for Michigan.

Access to care: Full NP scope of practice in Michigan would mean that within one or two years of adopting the policy, an estimated 580,000 additional adults (52 percent

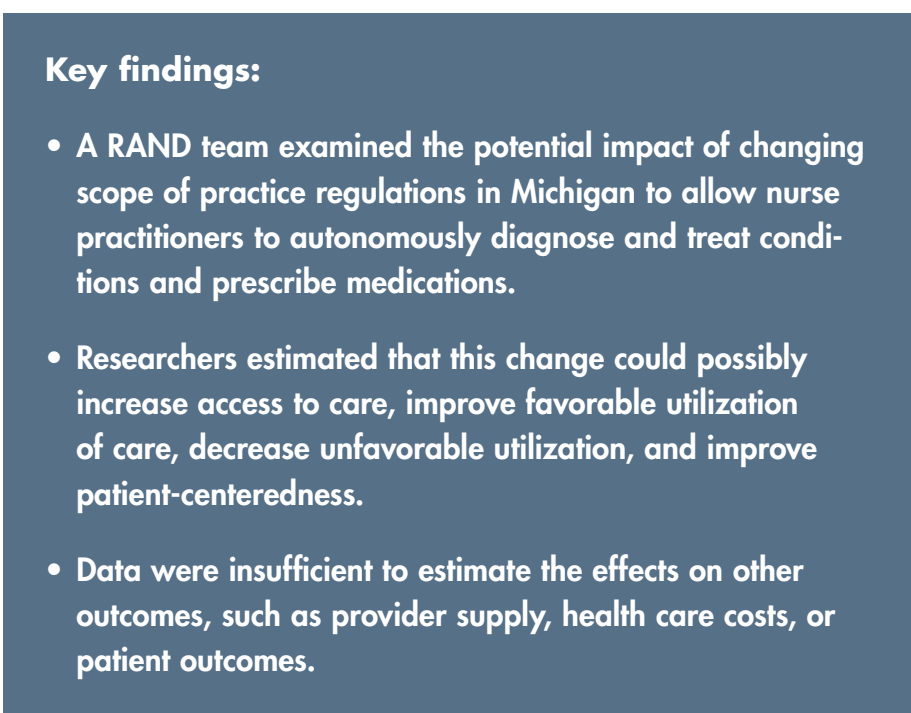

of adults, an increase of 7.5 percentage points) would be able to schedule a medical appointment when they wanted (see figure). Improvements in two other measures-ability to schedule an appointment when sick and ability to travel easily to a provider-increased by comparable amounts. The estimated effects were larger for children.

Utilization of care: Researchers estimated that full NP scope of practice would boost the number of provider-based office visits in Michigan by about 660,000 (a 3.1-percent increase over the baseline figure) and also increase the likelihood that adults would get an annual checkup by about 3.9 percentage points within two years of the change and by 6.8 percentage points after 11 years. Again, the effects were slightly larger for children. One study reported that full NP scope of practice reduced the number of ambulatory-caresensitive emergency department (ED) visits (that is, trips to the ED that could have been prevented by an office visit). These preventable ED visits would decrease by an estimated 36,000 (14 percentage points) within one or two years after

This research highlight summarizes RAND Health research reported in the following publication: Martsolf GR and Kandrack R, The Impact of Establishing a Full Scope of Practice for Nurse Practitioners in Michigan, Santa Monica, Calif.: RAND Corporation, RR-1639-MCNP, 2016 (available at www.rand.org/t/RR1639). 


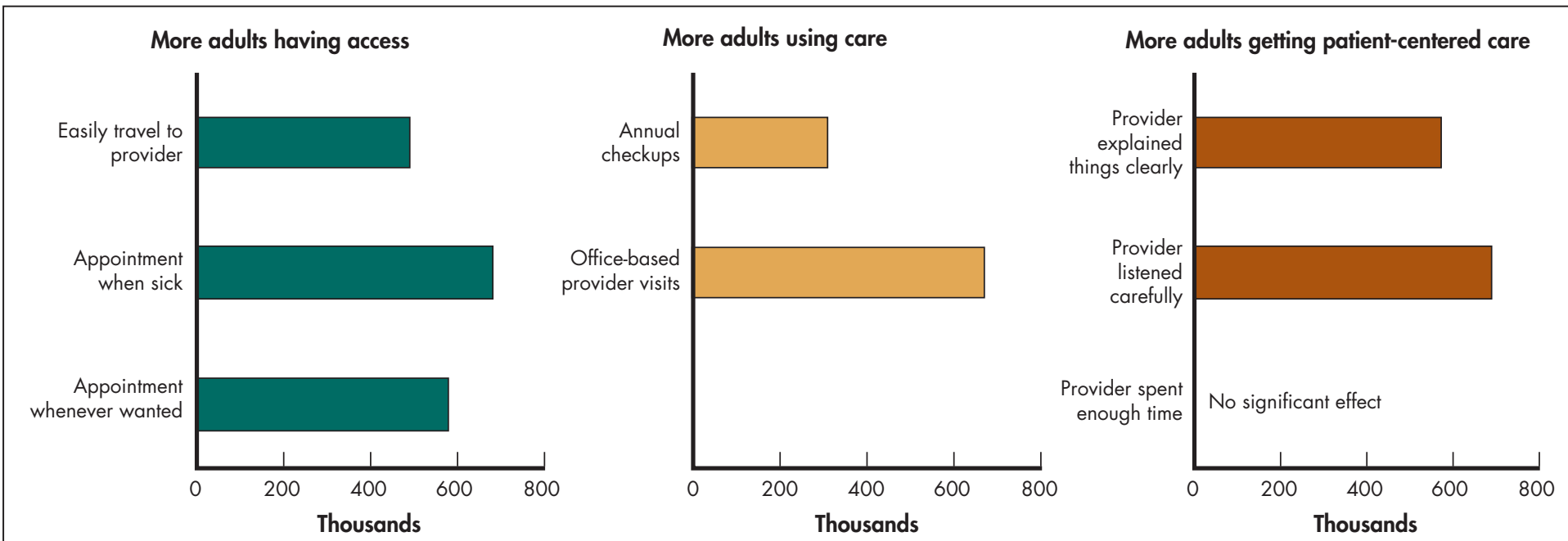

NOTE: Numbers represent estimated increases over baseline in Michigan 1-2 years after NP full scope of practice is established.

establishing full scope of practice and by 12.8 percentage points after 11 or more years.

Patient-centeredness: Patient-centeredness, an important dimension of quality of care, is the degree to which providers listen to and respond to patients and base clinical decisions on patient values. Full NP scope of practice has led to improvements in two aspects of patient-centered care in other states. Researchers estimated that in Michigan an additional 689,913 patients (an 8.9-percentage-point increase) would report that their provider listened carefully and that an additional 572,874 patients (a 5.4-percentage-point increase) would report that their provider explained things clearly. On a third measure-whether the provider spent enough time during the visit— the estimates showed no significant effect from full NP scope of practice.

The data on other dimensions of quality of care were not sufficient to allow reliable estimates of Michigan-specific effects.

Cost of care: Studies show a mixed relationship between full NP scope of care and costs. One national study found no effect on costs. Two others found that some components of costs - most notably NP wages - were lower in states with less-restrictive scope of practice rules. One found that the prices of well-child visits were lower in states with lessrestrictive scope of practice rules, though other prices were not affected. Researchers therefore characterized the effect of full NP scope of practice on costs as inconclusive. These results were not available in a form that allowed estimating specific impacts for Michigan.

Patient outcomes: Data were insufficient to estimate the effect of full NP scope of practice on patient outcomes.

\section{Conclusions and Implications}

The literature review found that granting NPs full scope of practice in Michigan could possibly improve access to care, utilization of care, and one dimension of quality - patientcenteredness. Given limitations in the evidence, researchers were unable to estimate the effect of a full NP scope of practice across a wide range of health care domains. The researchers also cautioned that because all of the studies examined had a national focus, their generalizability to the Michigan health environment carries a degree of uncertainty.

Further research is needed to assess the effects of expanding NP scope of practice on provider supply, costs, and other dimensions of health care quality.

This research was funded by the Michigan Council of Nurse Practitioners.

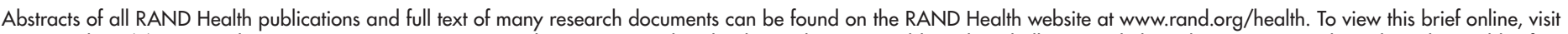

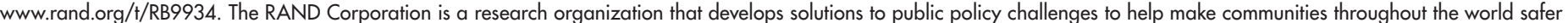

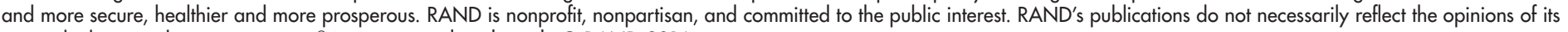
research clients and sponsors. RAND ${ }^{\circledR}$ is a registered trademark. @ RAND 2016.

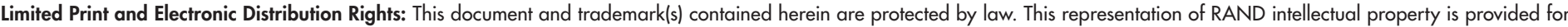

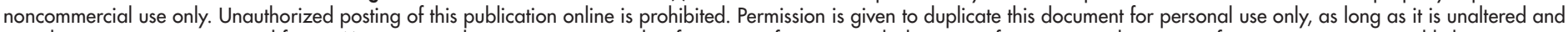

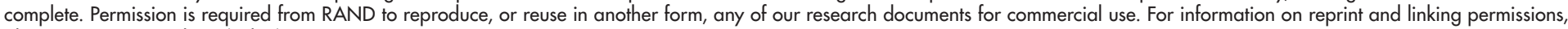
please visit www.rand.org/pubs/permissions. 